\title{
Onlineplattform „owidi“ bietet digitalen Zugriff auf wissenschaftlich fundiertes Wissen
}

\author{
Die Informations- und Wissensplattform owidi wird von der DGZMK (Deutsche Gesellschaft für Zahn-, \\ Mund- und Kieferheilkunde e. V.) in Kooperation mit Fachverlagen angeboten und hält qualifizierte \\ Inhalte in Form von Texten, Bildern, Grafiken und Filmen bereit. Zehn Minuten kostenlose Lesezeit wer- \\ den jedem neu registrierten Nutzer geschenkt.
}

$\mathrm{F}_{\mathrm{u}}^{\mathrm{i}}$ r Studierende der Zahnmedizin oder junge Zahnärztinnen und Zahnärzte ist der rasche und unkomplizierte Zugriff auf wissenschaftlich fundierte Fakten geradezu ein Muss. Hier bietet die DGZMK eine neue Möglichkeit, sich online schnell und verlässlich zu informieren: Die Informations- und Wissensplattform owidi (o-rales wi-ssen di-gital) ist ein neuer Service der DGZMK mit direktem Zugriff auf digitale Informationsquellen. Gemeinsam mit den DGZMK-Fachgesellschaften sowie den beteiligten Fachverlagen Deutscher Ärzteverlag, Dental Online College, Quintessenz Verlag und Springer Medizin bietet die DGZMK in dieser virtuellen Bibliothek qualifizierte Inhalte in Form von Texten, Bildern, Grafiken und Filmen zur Nutzung an. „Mit owidi haben wir einen Service geschaffen, der ganz neue Möglichkeiten im Umgang mit dem fachlichen Wissen in der Zahnmedizin eröffnet", ist DGZMK-Präsident Prof. Dr. Michael Walter überzeugt. „Testen Sie uns noch heute - wir freuen uns auf Sie!"

Wissen aus den unterschiedlichen Fachbereichen der Zahnmedizin lässt sich aus einer Vielzahl von Weiterbildungskursen oder Fachbüchern gewinnen. Eine Alternative dazu bietet owidi. Denn was in der Praxis oder im Studium oft fehlt, ist eine punktuelle, zeitnahe Entscheidungsunterstützung. owidi liefert schnellen Zugriff auf verschiedene Medien zu einem Thema (Bilder, fachliche Beiträge, Videos und Leitlinien), um kritische und komplexe Entscheidungen zeitnah treffen zu können oder sich einen Überblick zu verschaffen. Aber auch die wissenschaftlichen Leitlinien der DGZMK, die Profile der in ihr vereinten Fachgesellschaften und Arbeitskreise sowie die Inhalte der Mitgliederzeitschrift DZZ (Deutsche Zahnärztliche Zeitschrift) stehen zur Verfügung.

Mit der sogenannten „myQ-Suche“ lassen sich viele Inhalte der Verlage direkt recherchieren und finden. Die myQ-Suche ist das Herzstück von owidi, eine algorithmusgestützte Recherche, die, basierend auf dem jeweiligen Leseverhalten, Empfehlungen anzeigt. Die Suchergebnisse werden dadurch präziser und die Recherchezeit verkürzt sich messbar.

Jeder Nutzer, der sich neu auf owidi registriert, erhält zum Einstieg zehn Minuten Lesezeit geschenkt. So besteht die Möglichkeit, die Inhalte der Verlage zu testen. Die Registrierung ist kostenfrei. Dabei unterstützt der owidi-Registrierungsassistent jeden Neuankömmling effektiv bei der Einrichtung seines Benutzerkontos. Im Anschluss an die Registrierung erhalten Sie Zugang zum owidi-Reader, jenem Werkzeug, das Ihnen die Nutzung Ihrer Lesezeit ermöglicht. Ohne Registrierung ist nur ein eingeschränkter Zugriff auf die Inhalte innerhalb von owidi möglich. Damit auf sämtliche Inhalte und auf die geschützten Bereiche (z. B. Fachforum, geschlossene Bereiche innerhalb von Fachgesellschaftssuiten bei bestehender Mitgliedschaft in der entsprechenden Fachgesellschaft etc.) oder auf kostenpflichtige Inhalte zugegriffen werden kann, ist eine vorherige Registrierung erforderlich.

Exklusiv bietet owidi den Zeitleseservice „All You Can Read“. Um diesen nutzen zu können, ist das Installieren des owidiReaders vonnöten. Diese Software ist für Windows und Macintosh verfügbar. Anleitungen dazu finden sich auf der Website. Um „All You Can Read“ zu verwenden, muss zunächst Lesezeit erworben werden. Anschließend kann das gewünschte Dokument über den owidi-Reader aufgerufen werden. Dort wird dann „All You Can Read“ angeklickt. Entsprechend der Lesezeit öffnet sich das Dokument und kann gelesen werden. Abgerechnet wird dabei nur die Zeit, die tatsächlich für das Dokument oder den Film benötigt wurde.

Weitere Informationen und Hilfen zur Nutzung von owidi finden sich unter "Hilfe" auf www.owidi.de 